\title{
Cultural Adaptation of Conversational Style in Intercultural Computer-Mediated Group Brainstorming
}

\author{
Hao-Chuan Wang ${ }^{1}$, Susan R. Fussell ${ }^{2}$ \\ ${ }^{1}$ Information Science, ${ }^{2}$ Department of Communication \\ Cornell University \\ Ithaca, NY 14850 USA \\ [hw346, sfussell]@cornell.edu
}

\begin{abstract}
The ubiquity of international and intercultural collaboration over computer-mediated communication (CMC) raises questions about how culture, group cultural composition and medium influence communication. We conducted a laboratory study investigating the influences of individual cultural background (American versus Chinese), group cultural composition (same- versus mixed-culture groups), and communication medium (text-only versus videoenabled chatrooms) on the process of group brainstorming conversations. The paper presents an analysis of cultural adaptation focusing on whether and how individuals adapt their conversational behaviors when encountering group members from another culture. Chinese participants increased their responsiveness when working with American partners (i.e., mixed-culture groups). We also examine patterns of cultural adaptation by time and communication medium to gain insights for future research.
\end{abstract}

\section{Author Keywords}

Intercultural collaboration, computer-mediated communication, group brainstorming

\section{ACM Classification Keywords}

H5.3 Group and Organization Interface: Computersupported cooperative work

\section{INTRODUCTION}

The wide availability of computer-mediated communication (CMC) technologies makes it easy for geographically dispersed group members to perform teamwork remotely. Some recent research has shown interactions between culture and communication media on collaborative work (e.g., [2][8]). For example, Chinese pairs engage in more in depth conversations when rank ordering items than do American pairs, and these cultural differences are heightened when the pairs communicate face-to-face vs. via Instant Messaging [8].

Moving beyond the interaction effects between individual cultural background and medium, what remains

Copyright is held by the author/owner(s).

IWIC'09, February 20-21, 2009, Palo Alto, California, USA.

ACM 978-1-60558-198-9/09/02. underexplored is how individual cultural background and group cultural composition interact and influence remote collaboration. The literature on intergroup communication has found that individuals adapt their communicative behaviors, such as accent and language use, when conversing with people from other backgrounds (e.g., culture) [4][6]. In the context of computer-mediated intercultural collaboration and CMC more generally, it is of importance to understand whether and how adaptation of communication styles occurs, and to understand its implications for technology adoption and design.

Group brainstorming is a popular and important group task aiming at supporting creative idea generation through social means. As individuals are often limited in their visions or cognitive resources, group brainstorming is considered potentially helpful not only by pooling ideas from individuals but also by stimulating individuals to explore categories of ideas they fail to think about on their own [7]. Because group brainstorming aims to elicit creative, unconventional and even deviant ideas through social interaction, people's behavior during group brainstorming is affected by peer evaluation and social conformity [3]. This characteristic qualifies group brainstorming as an ideal arena for testing communication adaption because conversational moves (e.g., proposing ones own ideas, following others' ideas) may be viewed as a stylistic feature of communication shaped by evaluation pressure one perceives. Culture, group cultural composition and medium may influence perceived evaluation pressure and thus affect their communication styles.

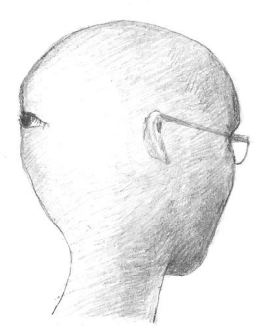

Figure 1. The "Extra Eye" brainstorming task used in our study. Participants were asked, "What are the benefits and difficulties if people had an third eye in the future?" 
In the current study, we examine how team members' national cultural background (American vs. Chinese), group cultural composition (same- versus mixed-culture groups), and communication medium (text-only vs. video-enabled chatroom) influence conversational styles during group brainstorming. We identified patterns of cultural adaptation in mixed-culture groups, such that participants with a Chinese cultural background adapted their communication styles and increased their responsiveness when working with American partners. We further explore how different media conditions influence cultural adaptation and how such patterns evolved over time.

\section{THEORETICAL BACKGROUND}

\section{Communication Accommodation}

Communication Accommodation Theory (CAT) posits that when individuals communicate with other people possessing distinct group memberships or social identities (e.g., culture, gender, power position in organizations), styles of communication may converge, diverge or be maintained [4][6]. Individuals may adapt various features of communication, ranging from features of vocalization such as accent and speech rate to non-verbal features such as gesture and posture, in order to accommodate a partner. Strategies at the level of discourse, including its planning and management, are more sophisticated strategies of accommodation to account for each other's specific conversational needs [4][6]. Discourse management is especially relevant to computer-mediated intercultural collaboration because (a) speech and non-verbal cues are often unavailable as tools for accommodation in CMC; and (b) the nature of intercultural collaboration is often taskand goal-driven, and thus how conversational threads are initiated and maintained is likely to directly influence the outcome of collaboration.

\section{Conversational Responsiveness}

Conversational responsiveness is a concept established in linguistic studies of dialogue structure [1]. Utterances in dialogues are often categorized as initiations versus responses. In everyday dialogues, initiations set up discourse expectations, and responses fulfill these expectations.

In group brainstorming conversations, however, an initiation (e.g., proposing an idea) does not necessarily call for any response because the purpose of the task is to generate as many ideas as possible but not to negotiate for consensus [5]. Decisions about whether responding to ideas proposed during brainstorming are made voluntarily as stylistic features of communication.

\section{Cultural Variation}

Cultural variation in attitudes and behaviors can be categorized and verified on various dimensions. Individualism-collectivism is often cited as one prominent distinction between Eastern and Western cultures. Individualistic cultures, such as those of the U.S., Canada and Germany, value the autonomy of individuals in groups and emphasize personal gain. Collectivistic cultures such as those of China and Japan tend to view individuals as components of the group and focus on group goals [9].

Individualism-collectivism is relevant to intercultural collaboration because it describes the motivational structures behind people's management of conversational moves. In a brainstorming task, individuals from collectivistic cultural backgrounds may be apprehensive to propose deviant ideas and they may be more susceptible to evaluation pressure from peers because they value group harmony over self-performance in idea generation. They may also avoid responding critically to, or expressing opinions about, others' ideas. In contrast, members of individualistic cultures, which value the expression of personal thoughts, may be quite willing to contribute to group brainstorming conversations and to critique others' ideas.

This leads to our first hypothesis about how American participants and Chinese participants converse with others sharing the same cultural norms in same-culture groups:

H1: Since group brainstorming does not require people to respond to proposed ideas, a conversational move of responding signifies the disclosure of personal opinions. In intra-cultural conversation, Chinese participants, as collectivists, will have a relatively low rate of responsiveness because the want to avoid such disclosure and because they predict that their Chinese partners will share the same conversational norms. Americans, as individualists, will have fewer concerns about disclosing personal opinions and will thus exhibit greater responsiveness to their partners' messages.

In mixed-culture groups or intercultural encounters, intergroup accommodation of communication styles may come into play:

H2: Chinese participants will adapt their conversational responsiveness. In intercultural groups, Chinese participants will have less concern about disclosure of personal opinions, and thus will be more responsive to partners' messages. In contrast, Americans, because of their tendency toward individualism, will be less likely to adapt their responsiveness to match that of a Chinese partner.

\section{THE EXPERIMENT}

\section{Design}

Three-person groups were asked to perform two structurally similar brainstorming tasks, one via a text-only chatroom and one via a video-enhanced chatroom. Audio was unavailable for both conditions. Four types of cultural group are formed: three Americans (AAA), three Chinese (CCC), two Americans and one Chinese (AAC) and one American and two Chinese (ACC). Group cultural composition was a between-subject manipulation. Media 
and brainstorming topics were within-subject manipulations and were counterbalanced.

\section{Participants}

Forty-two participants were recruited from a U.S. university and surrounding community. Among them, 22 participants were Americans living in the U.S. for more than 10 years with English as their first language. The remaining 20 participants were international students born in China $(85 \%)$ or Taiwan $(15 \%)$ whose first language was Chinese. The Chinese participants were all fluent or nearly fluent in English. Although they were all currently studying at a U.S. university, the majority had been in the U.S. less than 2 years. Participants were randomly assigned to experimental conditions. A total of 14 brainstorming groups were formed (3 AAAs, 3 CCCs, 5 AACs, and 3 ACCs).

\section{Materials}

Two brainstorming tasks of equivalent difficulty were created: the "extra thumb" question and the "extra eye" question. The extra thumb question asked participants to generate ideas about the benefits and difficulties for people having a theoretical extra thumb in the future. This task has been used in many previous brainstorming studies [3]. The extra eye question is a newly designed task that looking for ideas about the benefits and difficulties for people having an extra eye at the back of their heads in the future (see Figure 1).

\section{Procedure}

Participants were brought to the laboratory and instructed about the brainstorming topics and brainstorming rules. They were provided with four conventional brainstorming rules [7]: (a) the more ideas the better; (b) the wilder the ideas the better; (c) combination and improvement of ideas are sought; and (d) avoid evaluating others' ideas. Groups were given 15 minutes for each of the two brainstorming tasks (only the first 12 minutes were included in the analysis for control). Between tasks, we switched which version of the chatroom they were using (text only or video enhanced text). Participants worked by typing as audio is controlled to be unavailable all the time.

\section{MEASURES}

To measure responsiveness, we first coded conversational turns by applying a coding scheme consisting of seven categories. Five main categories relevant to this analysis are: ideation (proposing ideas), meta-strategy (strategizing and coordinating moves), response (questioning or commenting antecedents), (dis-)agreement (acknowledgement, consent or dissent), and explanation (explaining prior ideas). Two independent coders performed the coding task. Inter-coder reliability based on $6 \%$ of the data was satisfactory (Cohen's Kappa=.69).

Among the coding categories, two general types were identified. Ideation and meta-strategy are active codes featuring self-initiated contributions. On the other hand,

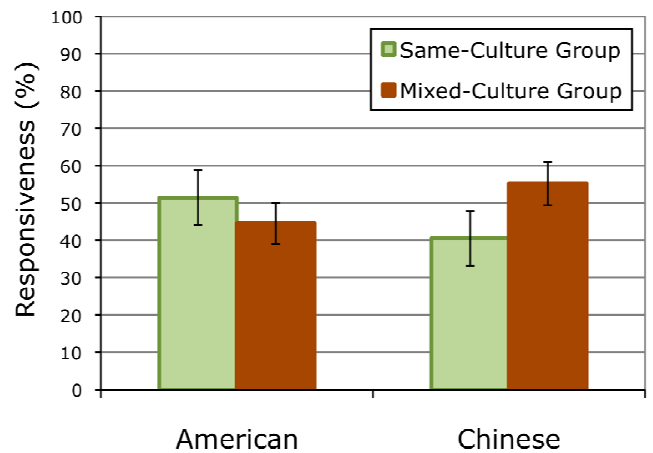

Figure 2. Responsiveness by individual cultural background and type of cultural group.

response, (dis-)agreement and explanation are reactive codes elicited by antecedents.

The measure of responsiveness is operationalized as the percentage of reactive utterances out of the total of active and reactive utterances:

\section{Responsiveness $=$ Number of Reactive Codes / (Number of Active Codes + Number of Reactive Codes)}

The higher the value, the more responsive a participant's messages are to the prior contributions by self or partners.

\section{RESULTS}

The hypotheses were investigated by using a mixed model ANOVA to account for possible local interdependency between data points caused by repeated measures and social interactions. Individual cultural background, type of cultural group (same-culture versus mixed-culture group) and their interactions were included as fixed effects in the model.

There was a marginal individual cultural background by type of cultural group interaction $(F[1,19.73]=3, p<.1)$. Consistent with $\mathrm{H} 1$, when working in same-culture groups, Americans were the most responsive (on average, $51.4 \%$ of their utterances were responsive) and Chinese were the least responsive (only $40.5 \%$ of their utterances were responsive) (see Figure 2).

In support of H2, when working in mixed-culture groups, participants raised their level of responsiveness $(55.2 \%$ of their utterances were responsive). Post-hoc tests revealed that the effect of cultural adaptation was marginal for Chinese participants (Chinese-Same Culture Group versus Chinese-Mixed Culture Group, $t=1.56, p=.14)$. Although from Figure 2 it appears that Americans lowered their responsiveness in intercultural context, this effect was not significant. Americans maintained the style.

\section{Temporal Dynamics and Medium Influence}

We took a closer look at cultural adaptation by breaking it down along temporal and medium dimensions. Figure 3 shows the three-way interactive patterns (culture, cultural groups and medium) for responsiveness over time. The 

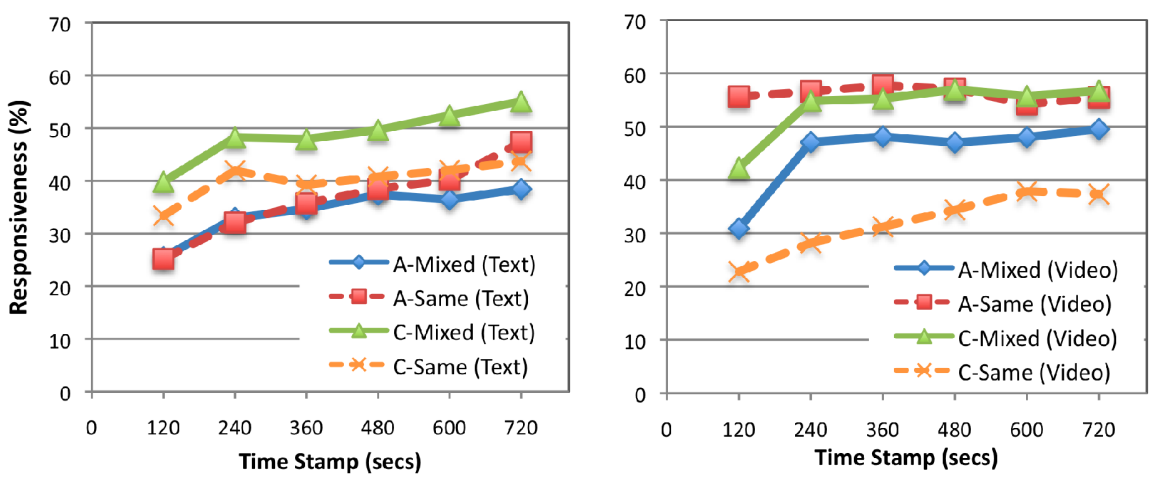

Figure 3. Responsiveness by culture, type of cultural group and medium over time.

Left: Text-only condition. Right: Video-enabled condition. (A=American, $C=C h i n e s e)$.

percentages of reaction codes were computed every two minutes (i.e., 120 seconds) until the end.

Although the graphs are complex, one initial observation is that medium seems to influence cultural variation and adaptation of communication styles. When video is available (Figure 3, right), cultural differences in intracultural contexts can be seen and the degree of cultural adaptation (e.g., the differences between Chinese-Same Culture and Chinese-Mixed Culture) is greater. In general, the text-only medium seems to have centralized and reduced cultural discrepancy.

It also appears that when visual cues were available, cultural adaptation occurred earlier in the interaction than when the cues were not available. In the video-enabled condition, at the beginning of the session (e.g., 120-second time stamp), both the Chinese and Americans already revealed strong adaptation tendencies. But in the text-only condition, cultural adaptation seemed to evolve over time. This suggests the pre-existence of knowledge of cultural adaptation and the prominence of non-verbal cultural cues in triggering such knowledge.

\section{DISCUSSION AND CURRENT DIRECTIONS}

If we only look at the overall interaction between cultural background and group cultural composition on responsiveness, it seems that Chinese participants adapted more toward American conversational styles than Americans adapted toward Chinese conversational styles. However, when the pattern is broken down by time and communication medium, Figure 3 suggests that Americans were adaptive, especially when visual cues were available.

Our results suggest that communication accommodation is a prominent phenomenon in computer-mediated intercultural collaboration. The adoption of communication technology seems to possess differential influences on group dynamics depending on cultural contexts.

The initial analysis is limited in its sample size. It is likely that continual coding and analytical efforts will help clarify the trend and patterns we saw.

\section{ACKNOWLEDGMENTS}

This material is based upon work supported by the National Science Foundation under Grants No. IIS-0803482, IIS0325047 and CNS-0551554. We thank Leslie Setlock for her assistance and comments.

\section{REFERENCES}

1. Carletta, J., Isard, A., Isard, S., Kowtko, J. C., DohertySneddon, G., Anderson, A. H. (1997). The reliability of a dialogue structure coding scheme. Computational Linguistics, 23, 13-31.

2. Diamant, E. I., Fussell, S. R. \& Lo, F-L. (in press). "Where did we turn wrong?" Unpacking the effects of culture and technology on attributions of team performance. Proceedings of CSCW 2008.

3. Dugosh, K. L., \& Paulus, P. B. (2005). Cognitive and social comparison processes in brainstorming. Journal of Experimental Social Psychology, 41, 313-320.

4. Giles, H., Coupland, J. \& Coupland, N. (1991). Contexts of Accommodation. NY: Cambridge University Press.

5. Jackson, M. H., \& Poole, M. S. (2003). Idea generation in naturally occurring contexts: complex appropriation of a simple group procedure. Human Communication Research, 29, 560-591.

6. Jones, E., Gallois, C., Callan, V., \& Barker, M. (1999). Strategies of accommodation: Development of a coding system for conversational interaction. Journal of Language and Social Psychology, 18, 123-152.

7. Osborn, A. F. (1957). Applied imagination. NY: Scribner.

8. Setlock, L D., Fussell, S. R., \& Neuwirth. C. (2004). Taking it out of context: collaborating within and across cultures in face-to-face settings and via instant messaging. Proceedings of CSCW 2004.

9. Triandis, H. C. (1995). Individualism and Collectivism. Boulder, CO: Westview. 